\title{
Clostridium Sporogenes Causing Bacteremia Originated from the Skin and Soft Tissue Infection in an Immunocompetent Patient - Case Report and Literature Review
}

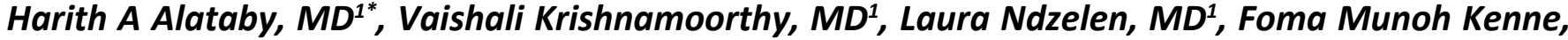 \\ $M D^{1}$, Kate Valenti, $M L T^{4}$, Jessie Savermuttu, $M D^{2}$ and Jay Nfonoyim, $M D^{1,3}$
}

\author{
${ }^{1}$ Department of Medicine, Richmond University Medical Center, New York, USA \\ ${ }^{2}$ Department of Infectious Disease, Richmond University Medical Center, New York, USA \\ ${ }^{3}$ Department of Critical Care, Richmond University Medical Center, New York, USA \\ ${ }^{4}$ Department of Microbiology, Richmond University Medical Center, New York, USA
}

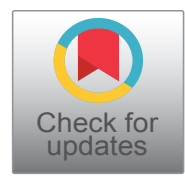

*Corresponding author: Harith Adnan Alataby, Department of Medicine, Richmond University Medical Center, Staten Island, New York, USA, Tel: 3463179648

\begin{abstract}
Introduction: Clostridium sporogenes is a major cause of food spoilage. In this report, we present a case of bacteremia in an immunocompetent patient, caused by $C$. sporogenes and most likely originated from the skin and soft tissue infections.

Case Presentation: 75-year-old man with PMHx of Parkinson's disease presented to ED after he was found lying on the floor inside his house, covered in urine and feces for unknown duration of time. He was extremely lethargic and dehydrated. He had BP90/60 mmHg, PR100, RR 22, temperature $100.5{ }^{\circ} \mathrm{F}$, and $\mathrm{SPO}_{2} 98 \%$ on room air. Skin examination revealed the presence of multiple pressure ulcers located on his face, chest, and left leg were covered with necrotic tissue and were unstageable. No abscess or gas gangrene was noted. In medical-ICU, he was treated for severe AKI (obstructive uropathy, rhabdomyolysis and dehydration), hypernatremia, hyperkalemia, lactic acidosis, leukocytosis and Bacteremia. Three sets of blood cultures were obtained; each of them collected two days apart. All three were positive for Clostridium sporogenes. The patient was treated with intravenous penicillin and vancomycin for seven days but was still having febrile episodes. Clindamycin was then added. His condition significantly improved and completed a 14-days. After this, the patient underwent surgical debridement of the necrotic pressure ulcers.
\end{abstract}

Discussion: Less than twenty-five cases of infections caused by $C$. sporogenes have so far been reported and include septic arthritis, pyogenic liver abscess, empyema, gas gangrene, and septicemia. To our knowledge, only seventeen cases of $C$. sporogenes bacteremia, including the one described in this case report, have ever been reported. Severe of them with Clostridium bacteremia had leukopenia. Fourteen out of these seventeen patients were diagnosed with malignancies. There were only three non-cancer patients with $C$. sporogenes bacteremia. A 45-year-old renal transplant recipient who developed acute empyema with hydropneumothorax treated aggressively with drainage and antibiotics, a 66-year-old immunocompetent patient with cellulitis and osteomyelitis of the lower limb, the patient refused limb amputation and ended up with death and the patient described in this case report who developed $C$. sporogenes bacteremia most likely due to infected pressure ulcers. First-line treatment is penicillin. Due to reported increased resistance of Clostridial species to Penicillin G and metronidazole, clindamycin, and cefoxitin combination antibiotic therapy may be required in certain patients who are infected with resistant strains of $C$. sporogenes.

Conclusion: Due to the high mortality rate associated with Clostridium bacteremia, early initiation antimicrobial therapy and surgical intervention are very important to control the source of infection, improve survival, and prevent complications.

\section{Keywords}

Clostridium Sporogenes, C. sporogenes, Clostridial infections, Anaerobic, Gram-positive bacillus, Bacteremia, Bloodstream infection

\footnotetext{
Citation: Alataby HA, Krishnamoorthy V, Ndzelen L, Kenne FM, Valenti K, et al. (2020) Clostridium Sporogenes Causing Bacteremia Originated from the Skin and Soft Tissue Infection in an Immunocompetent Patient Case Report and Literature Review. Int J Crit Care Emerg Med 6:095. doi.org/10.23937/2474-3674/1510095 Accepted: July 07, 2020: Published: July 09, 2020

Copyright: (C) 2020 Alataby HA, et al. This is an open-access article distributed under the terms of the Creative Commons Attribution License, which permits unrestricted use, distribution, and reproduction in any medium, provided the original author and source are credited.
} 


\section{Introduction}

The genus Clostridium comprises of over 200 described species that are associated with a variety of toxin-mediated as well as invasive infections, such as bacteremia, gas gangrene, foodborne botulism, and antibiotic-associated colitis. Clostridium sporogenes is one of the members of the genus Clostridium that is considered to be a significant cause of food spoilage. However, it is rarely recognized as a clinical pathogen. Less than 25 cases of infection secondary to this organism have been reported so far. In this report, we present a case of bacteremia in an immunocompetent patient, which was caused by $C$. sporogenes and most likely originated from the skin and soft tissue infections.

\section{Case Presentation}

A 75-year-old man with a past medical history of Parkinson's disease was brought to the emergency department of our hospital by ambulance after he was found lying on the floor in prone position inside his house. The patient most probably fell on the floor several days ago before he was found in this position. According to the next of kin, the patient had multiple falls in the past few months that were attributed by her to the patient's Parkinson disease. The patient did not seek medical attention for any of these episodes.

On arrival in the emergency department, the patient was found to be extremely lethargic and dehydrated. He had Blood Pressure 90/60 mmHg, Pulse Rate 100, Respiration rate 22 , Temperature $100.5{ }^{\circ} \mathrm{F}$, and $\mathrm{SPO}_{2}$ $98 \%$ on room air. On physical examination: Skin revealed the presence of multiple pressure ulcers located on his face, chest, and left leg that had developed from staying immobilized on the ground for several days. The ulcers were covered with necrotic tissue and were unstageable. No abscess or gas gangrene was noted to be present.

Initial Laboratory test result: WBC 16000/ $\mu \mathrm{L}, \mathrm{Hb} 14.1$ $\mathrm{g} / \mathrm{dl}, \mathrm{PLT} 129000 / \mu \mathrm{L}$, Na $153 \mathrm{mEq} / \mathrm{L}, \mathrm{K} 5.9 \mathrm{mEq} / \mathrm{L}$, Blood Urea Nitrogen 244 mg/dL, Creatinine 9.6 mg/dL, Glucose 138 , Creatine Kinase 599 U/L.

Initial Head/chest/abdomen/pelvis CT scans were unremarkable for relevant findings.

The patient was admitted to the medical intensive care unit (MICU) for eight days and was then transferred to the general medical floor, where he remained admitted for almost a month. In medical-ICU, he was treated for severe AKI (obstructive uropathy, rhabdomyolysis and dehydration), hypernatremia, hyperkalemia, lactic acidosis, leukocytosis and Bacteremia. Initially, in MICU, the patient was found to have a high white blood cell count and fever. Three sets of blood cultures were obtained; each of them collected two days apart. All three of the blood cultures were positive for Clostridium sporogenes. The patient was treated with intravenous penicillin and vancomycin for seven days but was still having febrile episodes. Then Clindamycin was added to the antibiotic regimen. The patient showed significant improvement in his condition and completed a 14-day course of each antibiotic. The skin debridement was postponed because of the unstable patient condition and the good response that had reached by medical management. After stabilization of the patient's condition, the patient underwent surgical debridement of the necrotic pressure ulcers. Biopsy and culture of the skin tissue were performed and did not reveal the presence of any pathogens.

\section{Discussion}

Members of the genus Clostridium are characterized as anaerobic, gram-positive, spore-forming rods that are found ubiquitously in soils and marine sediments, also as natural inhabitants of the gastrointestinal tract in humans and most other animals. These bacteria are well-recognized for their ability to cause serious and rapidly fatal diseases. The development of these diseases may be due to a variety of toxigenic or invasive processes [1].

Despite the presence of Clostridium species as a part of the natural intestinal microflora and in the outside environment, infection with these organisms is not common. This may largely be because of the anaerobic conditions that are required for the growth of these bacteria. Moreover, not all Clostridium species produce toxins to the same degree [2].

Clostridium sporogenes is one of the several described species that are included in the genus Clostridium. This anaerobic, gram-positive rod was first described in detail by Metchnikoff in the year 1908. The organism was isolated from the diarrheal material of patients who had chronic colitis as well as from the feces of normal individuals [3]. Clostridium sporogenes is a major cause of food spoilage, and due to its strong genetic and physiological similarity to Clostridium botulinum Group I, it is frequently used as a surrogate for this organism in illustrating the effectiveness of food preservation processes. Strains of both C. sporogenes and Group I C. botulinum exist in the environment as highly heat-resistant spores. This highly resistant dormant state allows these organisms to survive in hostile conditions, such as the absence of nutrients, desiccation, oxygen, high pressure, heat treatment, and toxic chemicals. The resistance properties of these Clostridial species are the primary reason why these organisms present serious food spoilage and food safety problem. However, the formation of botulinum neurotoxin may be used to differentiate Group I Clostridium botulinum from Clostridium sporogenes. C. botulinum is an extremely dangerous pathogen and is known to produce a highly potent neurotoxin, which results in a deadly 
neuroparalytic disease on ingestion. In contrast, $C$. sporogenes does not produce this neurotoxin and is rarely considered to be pathogenic $[4,5]$.

Regarding the isolation process of $C$. sporogenes, initially, it was isolated from anaerobic blood culture bottle, then to differentiate it from the other types of Clostridium, RapID ANA II system was used. RapID ANA II is a qualitative micro method utilizing standard and chromogenic substrates for the identification of medically important anaerobic bacteria isolated from human clinical specimens.

Less than 25 cases of infections caused by $C$. sporogenes have so far been reported and include septic arthritis, pyogenic liver abscess, empyema, gas gangrene, and septicemia. To our knowledge, only 17 cases of C. sporogenes bacteremia, including the one described in this case report, have ever been reported. Seven of the patients with Clostridium bacteremia had leukopenia, which demonstrates the significance of leukopenia in these patients. Another important finding was that 14 out of these 17 patients had malignancies. The only three non-cancer patients were a 45-year-old renal transplant recipient who developed acute empyema with hydropneumothorax that was followed by $C$. sporogenes bacteremia, a 66-year-old immunocompetent patient who developed $C$. sporogenes bacteremia secondary to cellulitis and osteomyelitis of the lower limb, and the patient described in this case report who developed $C$. sporogenes bacteremia most likely due to infected pressure ulcers [6-11].

Exotoxins produced by Clostridium species are thought to play a significant role in the pathogenesis of infections caused by these organisms. Clostridium sporogenes infection remains unknown; however, it seems to involve the production of a hemorrhagic toxin by the organism that has shown to cause tissue destruction in certain animal models. Vascular endothelial cells are the primary targets of this toxin. The hemorrhagic toxin has been found to have collagenase activity and serves to hydrolyze type III and IV collagens that are the major components of tunica intima and tunica media of blood vessels. Thus, the hemorrhagic toxin produced by $C$. sporogenes is an important virulence factor, and the hemorrhage caused by this toxin depends on its collagenase activity $[12,13]$.

Due to the high in-patient mortality rate associated with clostridium bacteremia, early diagnosis and prompt initiation of appropriate antimicrobial therapy are considered to be of great significance. This is particularly critical in patients with underlying conditions, such as alcoholism, necrosis of the small or large intestine, and intra-abdominal surgery. Urgent, thorough surgical debridement is also mandatory in the case of skin and soft tissue infections, as a means to control the source of infection, improve survival, and prevent complications [6].

In the absence of a rapid diagnostic test for this organism, early diagnosis is often difficult and results in the use of broad-spectrum empiric therapy. Several different types of antibiotics have been shown to be efficacious against Clostridium species in vitro or animal studies. These include penicillin, clindamycin, metronidazole, rifampin, tetracycline, chloramphenicol, and erythromycin. Penicillin $\mathrm{G}$ has historically been recommended for the treatment of clostridial infections. Currently, penicillin, in combination with clindamycin is widely used for the treatment of clostridial gas gangrene. The rationale behind the use of this combination of antibiotic therapy is that some strains of Clostridium may be resistant to penicillin but susceptible to clindamycin $[14,15]$.

Polymicrobial infection is very common in patients with Clostridium bacteremia, which means that blood cultures may frequently yield non-Clostridial microorganisms in addition to the Clostridium species. Therefore, until the results of bacteriological cultures are obtained, the empiric antibiotic therapy used should be broad-spectrum and active against Gram-positive organisms (e.g., penicillin or cephalosporin), Gram-negative organisms (e.g., cephalosporin, aminoglycoside, or ciprofloxacin), as well as anaerobic organisms (e.g., metronidazole or clindamycin).

Traditionally, the first-line treatment option for patients with confirmed Clostridium sporogenes infection is with penicillins [7]. A study performed by Bodey, et al. however, reported increased resistance of Clostridial species towards Penicillin $G$ as well as metronidazole, clindamycin, and cefoxitin [16]. Combination antibiotic therapy may, therefore, be required in certain patients who are infected with resistant strains of $C$. Sporogenes, as was the case with our patient who failed to respond completely to penicillin and vancomycin but responded rapidly to the addition of clindamycin to the treatment regimen.

\section{Conclusion}

Clostridium sporogenes is rarely a cause of clinically significant infection in humans. Medical literature has a paucity of case reports of $C$. sporogenes bacteremia. Hence, the identification of $C$. sporogenes as a cause of bacteremia in an immunocompetent patient made this an unusual case. Obtaining cultures and initiation of broad-spectrum empiric antimicrobial therapy promptly are critical in improving patient survival. Clostridium species remain susceptible to several antibiotics; however, combination antibiotic therapy may be required in certain cases. Surgical debridement is also required in the case of Clostridium bacteremia resulting from skin and soft tissue infections.

\section{Conflict of Interest}

The authors report no conflict of interest. 


\section{References}

1. Onderdonk AB, Garrett WS (2015) Gas gangrene and other clostridium-associated diseases. In: Bennett JE, Dolin R, Blaser MJ, Mandell, Douglas, and Bennett's principles and practice of infectious diseases. ( $8^{\text {th }}$ edn), Elsevier Churchill Livingstone, Philadelphia, PA, USA, 2768-2772.

2. Howard RJ (2010) Acute necrotizing cholangiohepatitis with clostridium perfringens: A rare cause of post-transplantation mortality. Gastroenterol Hepatol (NY) 6: 243-245.

3. Princewell TJT (1978) Differences in colony morphology and carbohydrate fermentation of Clostridium sporogenes. J Gen Microbiol 108: 315-319.

4. Brunt J, Cross KL, Peck MW (2015) Apertures in the Clostridium sporogenes spore coat and exosporium align to facilitate emergence of the vegetative cell. Food Microbiol 51: 45-50.

5. Brunt J, Plowman J, Gaskin DJ, Itchner M, Carter AT, et al. (2014) Functional characterisation of germinant receptors in Clostridium botulinum and Clostridium sporogenes presents novel insights into spore germination systems. PLoS Pathog 10: e1004382.

6. Abusnina W, Shehata M, Karem E, Koc Z, Khalil E (2019) Clostridium sporogenes bacteremia in an immunocompetent patient. ID Cases 15: e00481.

7. Shen DX, Babady NE, Chen R, Gilhuley K, Tang YW (2013) Septicaemia caused by Clostridium sporogenes: Two case reports and a literature review. Rev Med Microbiol 24: 81 83.

8. Inkster T, Cordina C, Siegmeth A (2011) Septic arthritis fol- lowing anterior cruciate ligament reconstruction secondary to Clostridium sporogenes; a rare clinical pathogen. J Clin Pathol 64: 820-821.

9. Quattrocchi G (1963) Rare case of pyo-gaseous abscess of the liver caused by Clostridium sporogenes-Bacterium pyocyaneum. Riforma Med 77: 288-291.

10. Corbett CE, Wall BM, Cohen M (1996) Case report: Empyema with hydropneumothorax and bacteremia caused by Clostridium sporogenes. Am J Med Sci 312: 242-245.

11. Malmborg AS, Rylander M, Selander H (1970) Case report: Primary thoracic empyema caused by clostridium sporogenes. Scand J Infect Dis 2: 155-156.

12. Hara-Kudo Y, Yamakawa Y, Kumagai S (1996) Purification and some properties of Clostridium sporogenes hemorrhagic toxin. Biochem Biophys Res Commun 227: 413-418.

13. Hara-Kudo Y, Ogura A, Noguchi Y, Kumagai S (1997) Characteristics of toxicity and haemorrhagic toxin produced by Clostridium sporogenes in various animals and cultured cells. J Med Microbiol 46: 270-275.

14. Yang Z, Hu J, Qu Y, Sun F, Leng X, et al. (2015) Interventions for treating gas gangrene. Cochr Database Syst Rev 12.

15. Wells CL, Wilkins TD (1996) Clostridia: Sporeforming Anaerobic Bacilli. In: Baron S, Medical Microbiology. ( $4^{\text {th }}$ edn), University of Texas Medical Branch at Galveston, Galveston (TX), USA.

16. Bodey GP, Rodriguez S, Fainstein V, Elting LS (1991) Clostridial bacteraemia in cancer patients. A 12-year experience. Cancer 67: 1928-1942. 